\section{TICKER}

Kunstlicht macht Spinat wertvoller Lässt man frischen Spinat bis zu neun Tage in einer rund um die Uhr beleuchteten Auslage (bei ca. $0^{\circ} \mathrm{C}$ ), wird er keineswegs zu welkem Biomüll. Das fluoreszierende Licht, das Supermärkte zur Ausleuchtung ihrer Gemüseregale verwenden, regt eine verstärkte Vitaminproduktion im Blattgemüse an. Nach neun Tagen nimmt der Folatgehalt im Spinat um 84 bis $100 \%$ zu, der Gehalt an Vitamin K um 50 bis $100 \%$. Auch die Vitamine $\mathrm{C}$ und $\mathrm{E}$ nehmen signifikant $z$. Wird das Gemüse nicht beleuchtet, sondern bei gleicher Temperatur im Dunkeln aufbewahrt, ändern sich die Vitaminkonzentrationen nicht oder nehmen leicht $a b$.

$\mathbf{R M}$ -

Journal of Agricultural and Food Chemistry, 2010; 58 (5): 2980 DOI: 10.1021/jfgo3596v

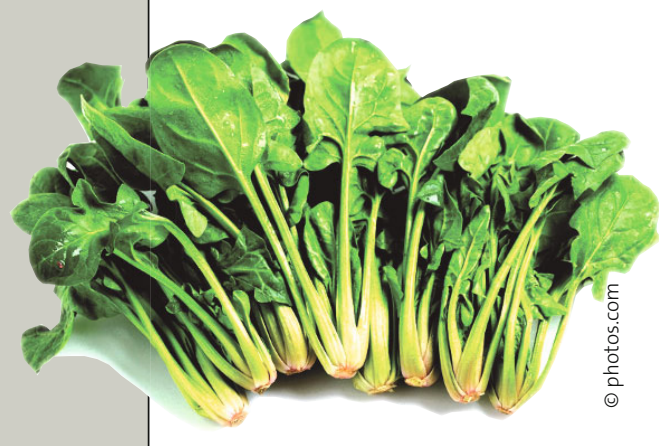

Riskante Gewichtsschwankungen Normalgewichtige Menschen haben offenbar nur dann ein günstigeres kardiovaskuläres Risikoprofil als übergewichtige Menschen, wenn sie ihr Gewicht einigermaßen konstant halten. Ein Auf und Ab beim Gewicht erhöht ihr Risiko in den Bereich von übergewichtigen Personen. Menschen, die zu viele Kilos auf die Waage bringen, müssen dagegen nicht fürchten, sich durch den Jo-Jo-Effekt einem zusätzlichen Risiko auszusetzen: Ihr ohnehin erhöhtes Herz-Kreislauf-Risiko wird durch Gewichtsschwankungen nicht weiter in die Höhe getrieben.

BS -

Waring ME et al. EPI/PNAM, 3.März 2010, San Francisco/CA, Abstract $P_{13} 8$

\title{
JOGGEN ODER WANDERN?
}

\section{Für die kardiovaskuläre Prävention ist das nicht entscheidend}

Wenn man durch Bewegung $1000 \mathrm{kcal}$ pro Woche verbraucht, spielt es dann eine Rolle, ob man dafür rennt oder nur schnell geht? Aus kardiovaskulärer Sicht macht es offenbar keinen großen Unterschied, wie eine Langzeit-Studie mit 43647 Probanden jetzt ergeben hat. Diejenigen, die bei hoher Belastungsintensität trainierten, hatten nur ein geringfügig geringeres Risiko für Herztod, Herzinfarkt oder Schlaganfall als Studienteilnehmer, die sich nur mäßig belasteten. Entscheidend war vielmehr der Energieverbrauch insgesamt: je höher, desto geringer die Gefahr von kardiovaskulären Komplikationen. Damit widersprechen die Studienergebnisse auch Befürchtungen, dass es einen
Umkehrpunkt gibt und extreme körperliche Belastungen wie etwa Marathonläufe dem Herz Schaden zufügen.

Für den Praxisalltag ergeben sich aus der Studie zwei Botschaften:

1. Man muss nicht unbedingt versuchen, aus Stubenhockern Sportskanonen zu machen. Es lohnt sich schon, wenn man solche Patienten zu ausgedehnten Spaziergängen motivieren kann.

2.Patienten, die bereits sportlich aktiv sind, können Sie ermuntern, ihre Aktivitäten noch auszudehnen, weil sie dadurch die positiven kardiovaskulären Effekte weiter steigern.

BS =

Chomistek AK et I. EPI/PNAM, 3. März 2010, San Francisco/CA, Abstract 2

\section{RISIKOFAKTOREN FÜR ASTHMA IM KINDESALTER}

\section{Aus für die Hygienehypothese?}

Die Hygienehypothese („zu viel Hygiene und wenig Fremdkontakte fördern kindliches Asthma“) wird durch neue Studiendaten infrage gestellt, berichtete Prof. Monika Grappa aus Wesel. Gegen die These spricht eine niederländische Studie mit fast 4000 Kindern, die von der Geburt bis zum achten Lebensjahr beobachtet wurden. Ein früher Krippenbesuch war mit vermehrten Atemwegsinfekten und einem erhöhten Risiko für obstruktive Bronchitiden im Vorschulalter verbunden. Der frühzeitige enge Kontakt mit anderen Kindern hatte jedoch keinen Einfluss auf Asthmasymptome, atopische Sensibilisierung und bronchiale Hyperreaktivität, so Grappa.

Im Gegensatz dazu kommt eine US-Studie mit fast 1000 Kindern zu dem Ergebnis, dass die Betreuung in Gemeinschaftseinrichtungen $a b$ dem zweiten Lebensjahr sehr wohl vor der Manifestation eines persistierenden Asthmas schützt. Rhinoviren haben als Triggerfaktoren von Asthma eine größere Bedeutung als RSViren, berichtete Grappa weiter. In einer

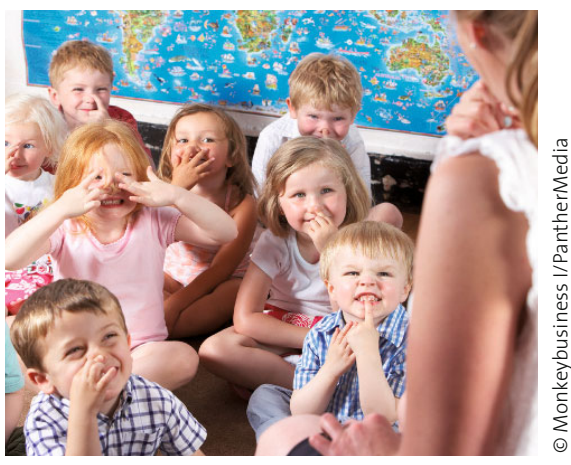

Schützt der Krippenbesuch vor Asthma? Die Datenlage ist widersprüchlich.

Studie war bei Rhinovirus-Infektionen das Asthmarisiko der Kinder fast zehnfach erhöht, bei vorangegangenem RSVInfekt nur um das 2,6-Fache.

Zu den Risikofaktoren für kindliches Asthma zählt nach neuen Daten außerdem häufiges Schwimmen in Freibädern mit chloriertem Wasser. Dagegen erhöht eine Antibiotikatherapie das Asthmarisiko nicht, wie neue Daten bestätigen.

PneumoUpdate, Wiesbaden, 20./21. November 2009 